\title{
Refined parcellation of the nervous system by algorithmic detection of hidden features within communities
}

\author{
Dongmei Shi, ${ }^{1}$ Anna Levina, ${ }^{1,2}$ and Hamid R. Noori ${ }^{1,3}$ \\ ${ }^{1}$ Max Planck Institute for Biological Cybernetics, Tübingen 72076, Germany \\ ${ }^{2}$ Department of Computer Science, University of Tübingen, Tübingen 72076, Germany \\ ${ }^{3}$ Courant Institute for Mathematical Sciences, New York University, New York 10012, USA
}

(Received 29 June 2018; revised manuscript received 14 May 2019; published 3 July 2019)

\begin{abstract}
The nervous system can be represented as a multiscale network comprised by single cells or ensembles that are linked by physical or functional connections. Groups of morphologically and physiologically diverse neurons are wired as connectivity patterns with a certain degree of universality across species and individual variability. Thereby, community detection approaches are often used to characterize how neural units cluster into such densely interconnected groups. However, the communities may possess deeper structural features that remain undetected by current algorithms. We present a scheme for refined parcellation of neuronal networks, by identifying local integrator units (LU) that are contained in network communities. An LU is defined as a connected subnetwork in which all neuronal connections are constrained within this unit, and can be formed for instance by a set of interneurons. Our method uses the Louvain algorithm to detect communities and participation coefficients to discriminate local neurons from global hubs. The sensitivity of the algorithm for discovering LUs with respect to the choice of community detection algorithm and network parameters was tested by simulations of different synthetic networks. The appropriateness of the algorithm for real-world scenarios was demonstrated on weighted and binary Caenorhabditis elegans connectomes. The detected LUs are distinctly localized within the worm body and clearly define functional groups. This approach provides a robust, observer-independent parcellation strategy that is useful for functional structure confirmation and potentially contributes to the current efforts in quantitative whole-brain architectonics of different species as well as the analysis of functional connectivity networks.
\end{abstract}

DOI: 10.1103/PhysRevE.100.012301

\section{INTRODUCTION}

The brain processes information as a multiscale network comprising local integrative components. Thus, a main focus of neuroscientists has been to subdivide brain into a mosaic of anatomically and functionally distinct, spatially contiguous areas (cortical areas and subcortical nuclei) as a prerequisite for understanding how the brain works. These areas differ from their neighbors in terms of microstructural architecture, functional specialization, connectivity with other areas, and/or orderly intra-area topographic organization [1-6]. Accurate parcellation provides a map of where we are in the brain, enabling an efficient comparison of results across studies and communication among investigators [5]. Moreover, understanding the functional architecture of the brain system has the potential to improve the diagnosis, prevention, and treatment of various neurological and mental illnesses. The overall brain architecture is thought to be composed of four systems - motor, sensory, behavioral state and cognitivewith parallel, distributed and (or) hierarchical subnetworks

Published by the American Physical Society under the terms of the Creative Commons Attribution 4.0 International license. Further distribution of this work must maintain attribution to the author(s) and the published article's title, journal citation, and DOI. within each system and complex, integrative interconnections between different systems. Specific groups of neurons with diverse anatomical and physiological properties populate each node of these sub- and supra-networks and form extraordinarily intricate connections with other neurons located near and far. Neuron connectivity forms the structural foundation underlying neural function.

Over the years, functional and structural maps of the nervous system in different animals have been established through the application of invasive (e.g., EGFP expression [7]) and non-invasive (e.g., dMRI [5]) methods. Numerous studies have charted connectomes of humans [6], macaques [8-10], rats [11], mice [7], Drosophila [12,13], and Caenorhabditis elegans [14-16]. These maps have been created on macro-, meso-, and microscales, their comprehensiveness and functionality are growing with the development of new techniques. In general, the neuroanatomical networks are hypothesized to be hierarchical and modular in different species [13,17-20].

Interneurons play a significant role in information processing in the neural system. In the striatum's internal microcircuit $[21,22]$, striatal interneurons integrate signals from diverse cortical areas and are capable of influencing the output of the circuit, whereas striatal projection neurons are affected by inhibition from interneurons; meanwhile, they are the main targets of cortical projections, and their axons originate pathways of the basal ganglia [23]. Thus, interneuron function appears to be closely related to information processing, whereas 
projection neurons are largely involved in information transfer. In our work, we presume that interneurons form a local integrator unit (LU) to perform information processing in the neural system, and we propose a network-theoretical approach to detect them. C. elegans is currently one of the only two organisms with a completely mapped connectome [24,25]. Hence, numerous studies have investigated the network-theoretical properties of the $C$. elegans connectome [14,26-31] and could demonstrate that analysis of its properties may lead to overarching general principles for higher species as well. Therefore, in addition to comprehensive tests of algorithmic performance on synthetic networks, we apply our method to the $C$. elegans nervous system to extract the LUs from neuroanatomical networks based solely on connectivity data to decipher its structure-function relationships. Our method provides a robust algorithm to detect LUs associated with distinct physiological and behavioral roles. Furthermore, it represents a simple framework to improve our understanding of recent findings [32,33] on the function of C. elegans neurons.

\section{METHODS}

\section{LU detection algorithm}

An LU is defined as a connected subnetwork of a neuronal community consisting of interneurons (INs) whose nerve fibers are completely constrained to that community. Furthermore, an LU must be connected to at least one other LU by a projection neuron. In a synthetic network, an ideal LU corresponds to a subgraph satisfying the following conditions:

(1) An LU is a connected induced subgraph.

(2) There are no direct edges between nodes from different LUs.

(3) There are no nodes in the network that could be added to one of the LUs forming a new LU that still fulfills the first two conditions (maximality condition).

Nodes belonging to this subgraph are called LU nodes, whereas nodes that do not belong to any LUs are called projection nodes or projection neurons (PN). We detect LUs by searching for such subgraphs in the neural network based on the $C$. elegans nervous system. Then, the question arises regarding how to confirm LUs in a network precisely. The entry point is the community structure.

Let us perform community detection in a network. This allows to determine a participation coefficient $P$ [34] of a node defining how this node is positioned in its own community and with respect to other communities. It is close to 1 if edges of the node are uniformly distributed among all communities and 0 if all its edges are within its own community. Therefore, the participation coefficient allows us to classify all nodes in a network into two groups: local nodes $(P=0)$, whose links are restricted to a single community, and nonlocal nodes $(P>0)$, with links in different communities. Figure 1 shows an example of a network consisting of communities. There are three communities in the grey shadows. According to the description of $P$, nodes in the network are classified into two types: local nodes in red with $P=0$ and nonlocal nodes in black with $P>0$. We can easily verify that the local nodes together with their interconnection in each community

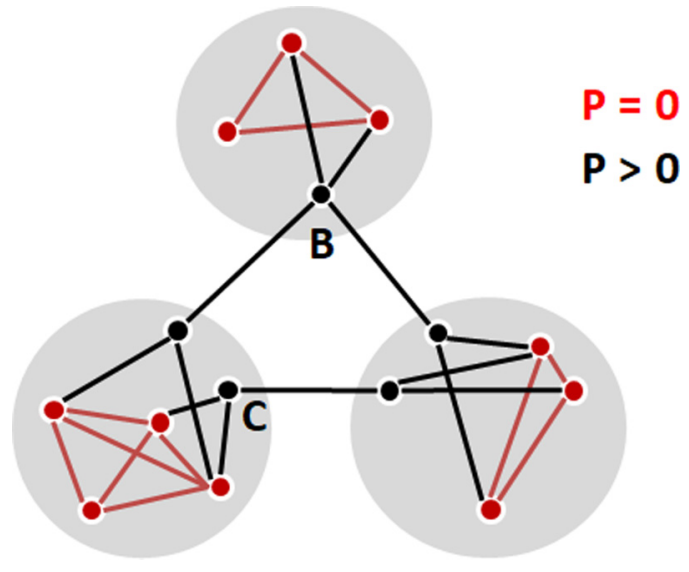

FIG. 1. Schematic illustration of community structure and local integrator units (LU). The three dark shadows represent three communities. The subnetworks in red indicate LUs, each red node represents an interneurons, and nodes in black such as $\mathrm{B}$ and $\mathrm{C}$ represent nonlocal nodes or projection nodes/neurons with links distributed among different communities $(P>0)$.

meet the conditions of being a LU if all nonlocal nodes are projection nodes. Therefore, finding local nodes is the main task of our work.

Based on the above analysis, we obtain LUs in a network by simply removing all nonlocal nodes. However, if the community structure is not distinctive, simultaneous removal can corrupt the detection of LUs. In addition, nonlocal nodes do not always correspond to projection nodes but may also be LU nodes that have connections with the projection nodes. For example (see Fig. 1), if nodes B and C are the two projection nodes in this network, then all other nodes together, with their inter-connectivity in each community, still meet the conditions of being LUs. Accordingly, the nonlocal nodes in black, excluding B and C, are also LU nodes. Considering that a nonlocal node with a higher $P$ is normally a projection node with a larger probability, we adopt a sequential algorithm: at each step, we remove only one nonlocal node with the maximum $P$. Our method therefore consists of the following steps:

(1) We detect the community structure in a network $G(V, E)$ by the Louvain algorithm (LA) [35] and use modularity $Q$ [36-39] as a quality function to evaluate the community division:

$$
Q=\frac{1}{2 m} \sum_{i, j}\left(A_{i j}-\frac{k_{i} k_{j}}{2 m}\right) \delta_{C_{i}, C_{j}},
$$

where $A$ is the adjacency matrix; $k_{i}$ and $k_{j}$ are the weighted degrees of the nodes $i$ and $j$, respectively; $m$ is the sum of all edge weights in the network; $c_{i}$ and $c_{j}$ are the communities of the nodes; and $\delta$ denotes the Kronecker $\delta$ function. Using LA to acquire community divisions, we obtain a participation coefficient $P_{i}(i \in 1, \ldots, \Lambda)$ for each node, where $\Lambda$ is the number of nodes in the network. A strict definition is given by

$$
P_{i}=1-\sum_{j=1}^{N_{c}}\left(\frac{k_{i j}}{k_{i}}\right)^{2},\left(0 \leqslant P_{i}<1\right),
$$




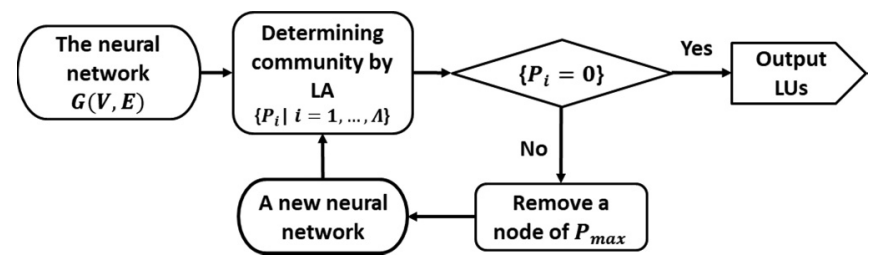

FIG. 2. Algorithmic flowchart of the LU detection technique. $P_{i}$ is the participation coefficient of node $i$, and $P_{\max }$ denotes the maximum participation coefficient.

where $N_{c}$ is the number of communities, and $k_{i j}$ is the number of links between node $i$ and all nodes in community $C_{j}$.

(2) We remove a node with maximal $P(>0)$ from this network sequentially. Then, we repeat community detection by LA and perform a removal until all nodes meet $\left\{P_{i}=0 \mid\right.$ $i \in \Lambda\}$.

(3) All communities are isolated from one another, and different communities correspond to different LUs.

The algorithmic flowchart of the method is shown in Fig. 2.

At this stage, it is necessary to discuss the differences and relations between the community structure (Com) and LU.

(1) In contrast to communities, a decomposition of a network into LUs does not lead to its full coverage. In other words, the network is divided into LU and nontrivial nonLU groups; whereas the network is a distinct union of its communities, i.e., $\forall G$

$$
\bigcup_{i}\left(\mathrm{LU}_{i}\right) \subset G
$$

and

$$
\bigcup_{j}\left(\operatorname{Com}_{j}\right)=G
$$

(2) Network communities are potentially overlapping, whereas LUs represent disjoint subgraphs of the network.

(3) With regard to the relations between LUs and communities, detecting an LU is like peeling the skin of communities and revealing the core. A community may contain more than one LU but may also contain no LUs.

LU structures can be found in many systems. For example, in the Drosophila brain, the local processing units are similar to LUs. The local units consist of interneurons and are proven to be important components of functional modules [12,13]. In the central processing unit, the arithmetic-logical units (ALU) are similar to LUs performing the actual arithmetic operations, and all operations of ALU are in the direction of the control units, which transmit coordinated control signals and commands. In a company, different departments have their own functions and are coordinated by the decision-making section of this company. In the human body, different organs perform different functions and are regulated by nerves and hormones to maintain interactions with each other. Therefore, our methodology provides insight into understanding a complex system and could be applied extensively to any system that has an LU structure.

\section{PERFORMANCE OF LU DETECTION TECHNIQUE ON SYNTHETIC NETWORKS}

\section{A. Performance evaluation function $S(C, D)$}

To verify the effectiveness of our algorithm, we apply the method to examples of synthetic networks with a predefined LU structure and calculate a score function $S(C, D)$ to quantify the algorithmic accuracy in predicting LUs. To this end, we define a synthetic network $G(V, E)$ according to the following sequence of steps:

(1) We initiate $G(V, E)$ to consist of $N$ connected components $\mathrm{LU}_{i}\left(V_{i}, E_{i}\right), i \in\{1, \ldots, N\}$. In each LU, every node connects with other nodes with an equal probability $(p, 0<$ $p \leqslant 1) . n_{i}$ denotes the number of nodes in $\mathrm{LU}_{i}$; therefore, $n_{i}=\left|V_{i}\right|$, and the average degree $\bar{k}_{i}$ in the $i$ th $\operatorname{LU}_{i}\left(V_{i}, E_{i}\right)$ is given by $\overline{k_{i}}=\frac{\left|2 E_{i}\right|}{n_{i}}$. We define $\bar{k}=\frac{\sum_{i=1}^{N} \overline{k_{i}}}{N}$, the average degree of all LUs. In the following we take $n_{1}=\ldots=n_{N}=n$.

(2) We add new $M$ nodes at one time to serve as projection nodes. Each new node, $j \in\{1, \ldots, M\}$, has $m_{j}$ edges randomly connecting with the nodes in the union of all LUs. We generate $m_{j}$ independently from the uniform distribution on $[2, \alpha]$ with $\alpha \in \mathbb{N}$ and $\alpha \geqslant 2$. To ensure the preservation of the LU structure, we prevent all edges from a projection node from being connected to the same LU.

There are five parameters that govern the structure of $G(V, E): n$ (number of nodes of LU), $N$ (number of LU), $\bar{k}$ (average degree of LUs), $M$ (number of projection neurons), and $\alpha$ (maximum out-degree of projection neurons). Figure 3 shows an example of $G(V, E)$ with three LUs (in blue) and ten projection nodes (larger size in red).

To define the score function $S(T, U)$, let us consider $T\left(V^{T}, E^{T}\right)$ as a subgraph of $G(V, E)$, which is partitioned into components:

$$
T\left(V^{T}, E^{T}\right)=\bigcup_{i=1}^{N_{T}} T_{i}\left(V_{i}^{T}, E_{i}^{T}\right),
$$

where $N_{T}$ is the number of components in $T$, and for any $i \in$ $\left\{1, \ldots, N_{T}\right\}, T_{i}$ is a connected induced subgraph of $G(V, E)$. In addition, for any $i \neq j, V_{i}^{T} \cap V_{j}^{T}=\emptyset$. We denote the set of all

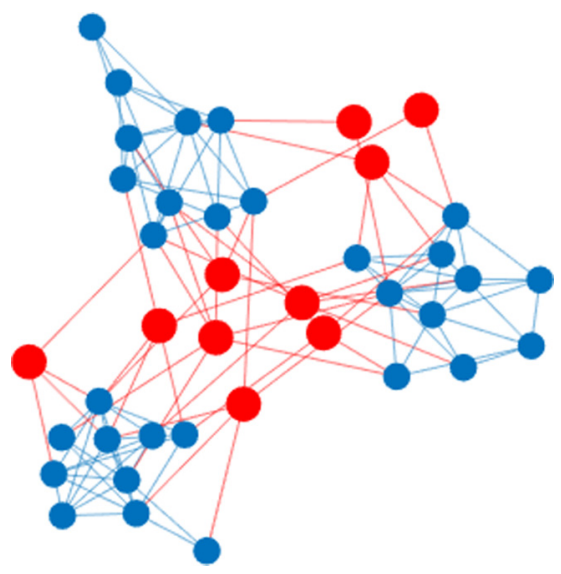

FIG. 3. An example of $G(V, E)$. There are three LUs $(N=3)$ with nodes and edges in blue. $n=10, \bar{k}=5, M=10, \alpha=7$. The projection nodes are marked by large red circles, and their connections are in red. 


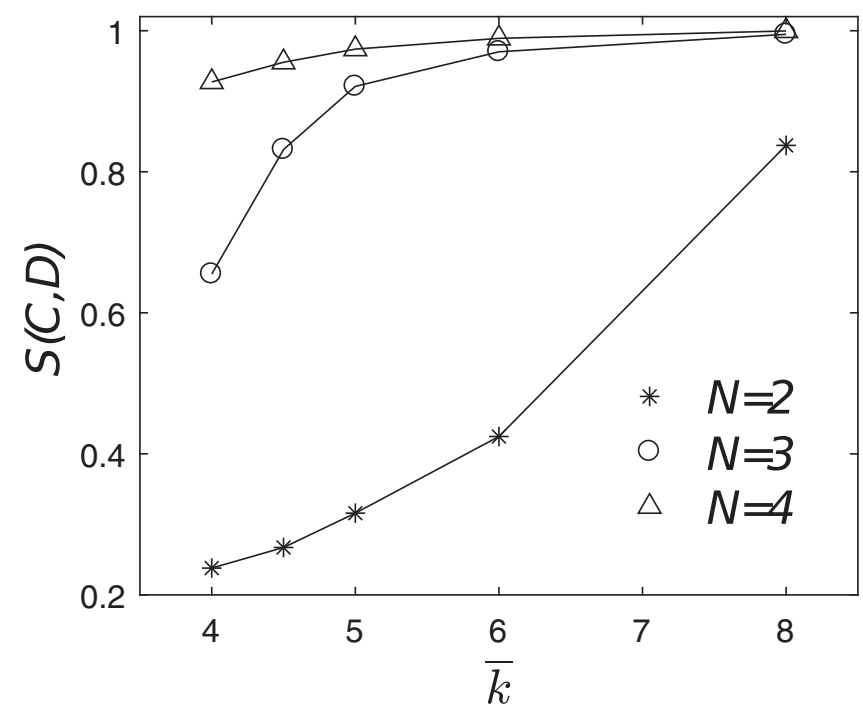

FIG. 4. Dependence of $S(C, D)$ on average degree $\bar{k}$. Stars: $N=$ 2, circles: $N=3$, triangles: $N=4$. Other parameters in both cases $n=20, \alpha=10, M=30$.

partitioned subgraphs of $G(V, E)$ by $P_{G}$. For any $T, U \in P_{G}$, we define a similarity relation $S: P_{G} \times P_{G} \rightarrow \mathbb{R}$,

$$
S(T, U):=\frac{1}{N_{T}} \sum_{i=1}^{N_{T}} \max _{j=1, \ldots, N_{U}}\left\{\frac{\left|V_{i}^{T} \cap V_{j}^{U}\right|}{\left|V_{i}^{T} \bigcup V_{j}^{U}\right|}\right\} .
$$

This function measures the ratio of the components of $T$ that are covered by the components of $U$. Thus, it provides an appropriate score function to evaluate the performance of our algorithm. We have $S(T, U)=1$ if and only if for each $i \in\left\{1, \ldots, N_{T}\right\}$, there exist $j \in\left\{1, \ldots, N_{U}\right\}$ such that $T_{i}\left(V_{i}^{T}, E_{i}^{T}\right)=U_{j}\left(V_{j}^{U}, E_{j}^{U}\right)$.

To demonstrate the accuracy and sensitivity of our algorithm, we evaluate its performance on artificial networks created by the steps as described in the previous section (e.g., in Fig. 3). We denote the built-in LU partition as $C=$ $\bigcup_{i=1}^{N} \mathrm{LU}_{i}\left(V_{i}, E_{i}\right)$. The detected structures create a subgraph $D, D=\bigcup_{j=1}^{N_{D}} D U_{j}\left(V_{j}^{\prime}, E_{j}^{\prime}\right)$, where $D U_{j}$ is the $j$ th unit detected by LU algorithm. We measure the performance quality of the algorithm by using the score function $S(C, D)$. Therefore, $S(C, D) \rightarrow 1$ indicates that the LU structure is detected correctly.

\section{B. Dependence of $S(C, D)$ on the number of LUs $(N)$ and the average degree of $\mathrm{LU}(\bar{k})$}

Figure 4 illustrates the dependence of $S(C, D)$ on $\bar{k}$ for $N=2,3,4$, while the remaining parameters are kept constant. We observe that $S(C, D)$ follows a power law and grows as the average LU degree $(\bar{k})$ increases, which means that denser intrinsic connections of LU lead to more efficient detection. Furthermore, the LU detection accuracy is directly proportional to the number of LUs $(N)$ in the network. It is also noted that $S(C, D)$ is much bigger at $N=3$ and 4 than that at $N=2$ (the minimal number of LUs in a network), and it rapidly increases to 1 as $\bar{k}$ increases.

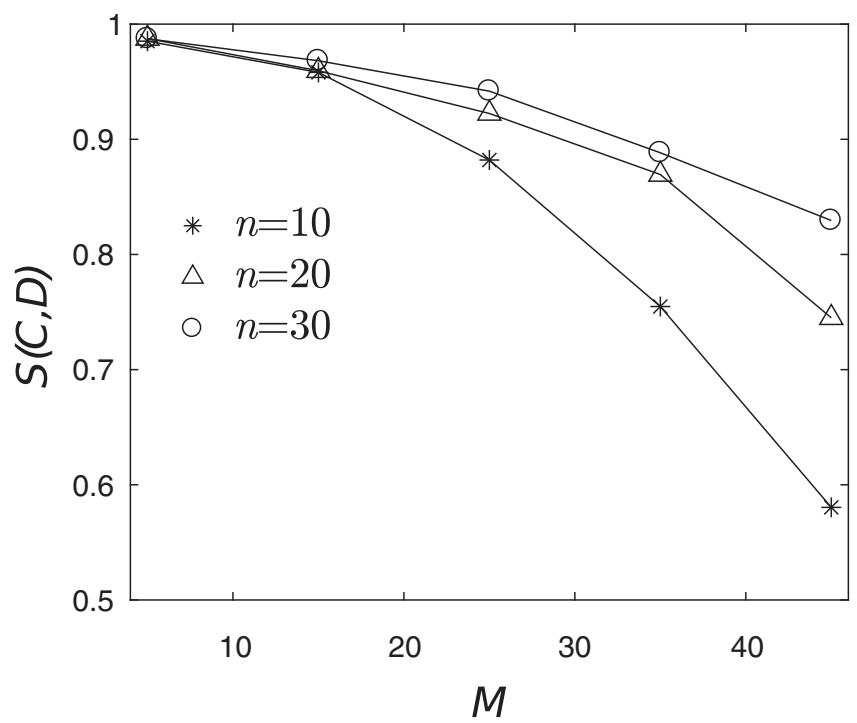

FIG. 5. Dependence of $S(C, D)$ on the number of projection nodes $M$. Star: $n=10$, triangles: $n=20$, circles $n=30$. Other parameters in all three cases: $\alpha=5, \bar{k}=5$, and $N=3$.

\section{Dependence of $S(C, D)$ on number of projection nodes $(M)$ and the size of LUs (n)}

The accuracy of the algorithm with respect to the number of projection nodes $M$ is simulated in Fig. 5. Each curve corresponds to $G(V, E)$ with an LU of different size $n$. Our findings suggest that $S(C, D)$ is monotonously decreasing in $M$. Thus, a large number of projection nodes is indicative of a less favorable LU detection by our algorithm. Moreover, LUs with a higher number of nodes are more likely to be detected. For instance, it is also observed that when $n=10$ and $M=40, S(C, D) \approx 0.70$. This means that even when the number of projection nodes is larger than the total number of nodes over all LUs $(40>10 \times 3)$, a large percentage of LU information can still be detected.

\section{Dependence of $S(C, D)$ on the maximum out-degree of projection nodes $(\alpha)$}

The left panel of Fig. 6 illustrates the dependence of $S(C, D)$ on $\alpha$ for $\bar{k}=4$ and 5 , respectively. It shows that as $\alpha$ increases, $S(C, D)$ increases to a plateau, while modularity $Q$ decreases monotonously. It also shows that a larger number of projection connections $\alpha$ increases the accuracy of LU detection only up to a $k$-specific threshold while blurring the community structure. The intuition behind this effect is that increasing $\alpha$ leads to an increase of average projection node degree. This in turn increases the participation coefficient [Eq. (2)] of projection nodes making them easier to detect. For a large $\alpha \gg \bar{k}$ the probability to remove the projection node is not increasing any more, generating an observed plateau.

In summary, we conclude that our algorithm detects the LU structure very efficiently, with the exception of one situation in which $S(C, D)<0.5$ when the number of LUs is the minimum, i.e., $N=2$ (see Fig. 4). For other cases, $S(C, D)$ remains much larger than 0.5 even at a very low connection degree $\bar{k} \simeq 5$ during all tests. In reality, LUs in most complex systems have a much denser intrinsic connection degree $\bar{k}$ and 

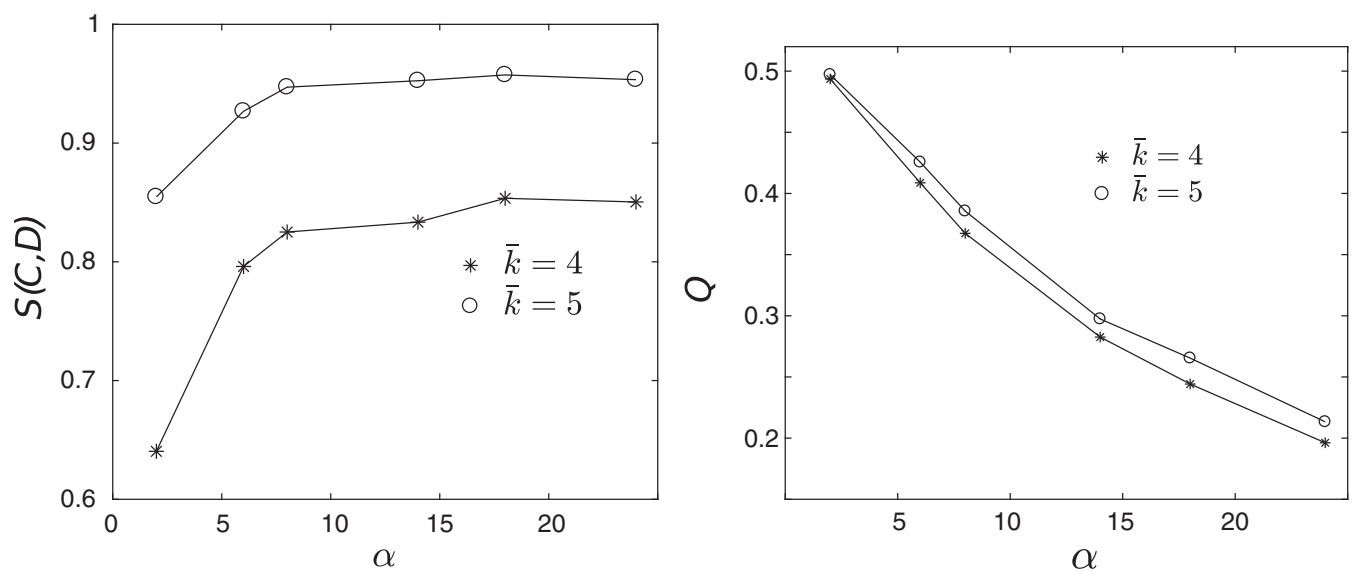

FIG. 6. (Left) Dependence of $S(C, D)$ on $\alpha$, and (Right) Dependence of modularity $Q$ on $\alpha$ for $\bar{k}=4$ and 5 , respectively. Stars: $\bar{k}=4$, circles, $\bar{k}=5$. Other parameters in both cases $n=10, N=3, M=20$.

a larger size, which ensures the better adaptability and higher efficiency. In addition, the tests of $S(C, D)$ for the connection density $\alpha$ further verify the robustness of the procedure. The simulations suggest particularly that not the number of projection nodes but rather their efficacy in connecting LU is critical for a good performance of the algorithm.

\section{E. Sensitivity with respect to choice of community detection algorithm}

The efficiency arguments suggest that it is reasonable to adopt Louvain algorithm for the community division step of LUs detection. However, considering that many different community detection methods have been proposed [34-38, 40-47], it is critical to evaluate the performance of our algorithm with respect to the choice of techniques. For this purpose, we calculated and compared the performance scores $S(C, D)$ of our model for two community detection algorithms, namely the modularity-based Louvain algorithm (LA) and the widely used Newman algorithm (NM) [36,38]. The latter detects communities by a spectral modularity maximization algorithm. In particular, we evaluated the dependencies of $S(C, D)$ on $\bar{k}$ (Fig. 7) and $\alpha$ (Fig. 8) using NM, and compared them with the results of LA.

The simulations confirm that the performance $S\left(C, D_{\mathrm{NM}}\right)$ of our model with Newman algorithm based community detection (as of $S\left(C, D_{\mathrm{LA}}\right)$ with LA-based detection) grows as $\mathrm{LU}$ average degree increases, and it is directly proportional to the number of LUs $(N)$. The results for both methods are qualitatively similar, although for $N=2, S\left(C, D_{\mathrm{NM}}\right)>$ $S\left(C, D_{\mathrm{LA}}\right)$, whereas for $N>2$, the performance of both algorithms is equivalent, Fig. 7. However, dependence of $S\left(C, D_{\mathrm{NM}}\right)$ and $S\left(C, D_{\mathrm{LA}}\right)$ on $\alpha$ suggests a superior performance of Louvain algorithm (Fig. 8). Since in the nervous system, most projection neurons have a large out-degree and connect diversely with various structures, i.e., $\alpha \gg 0$, it can be concluded that the integration of LA in our algorithm is more appropriate for applications in neuroscience. This statement is further supported by the following features of the Louvain algorithm: (1) LA finds high modularity partitions of large networks in a short time. (2) LA unfolds a complete hierarchical community structure for a network. Thereby, it gives access to different resolutions of community detection. As $\alpha$ is a hierarchical parameter, this capability of Louvain may also contribute to it producing a better starting point. (3) The resolution limit problem of modularity can be circumvented to a large extent by applying LA. Modularity optimization often fails to identify communities smaller than a certain scale, thereby inducing a resolution limit on the community detected by a pure modularity optimization approach [35]. Louvain algorithm overcomes this issue by displacing single nodes from one community to another. Thus, the probability that two distinct communities can be merged by moving nodes one by one is very low. (4) the ordering of the nodes does not have a significant influence on the results of LU detection.

\section{F. Multiple LUs within a community}

Our algorithm shows robust capability to detect local integrator units and parcellate the networks accordingly. However,

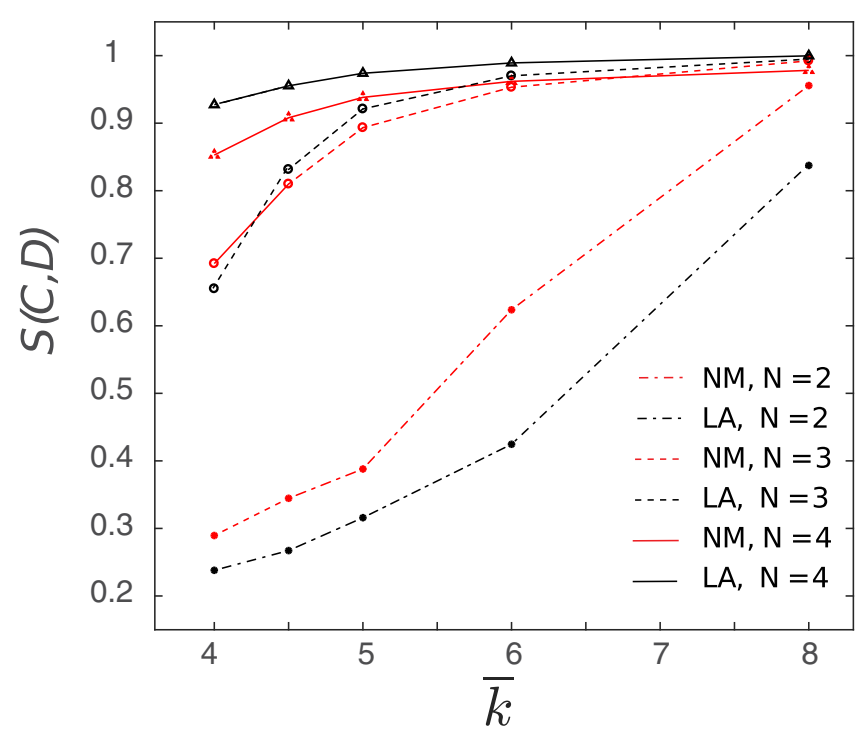

FIG. 7. Dependence on average degree $\bar{k}$ is qualitatively equivalent for both algorithms. Red symbols and lines correspond to Newman algorithm, black symbols represent Louvain algorithm. Stars: $N=2$, circles: $N=3$, triangles: $N=4$. Other parameters in both cases $n=20, \alpha=10, M=30$. 


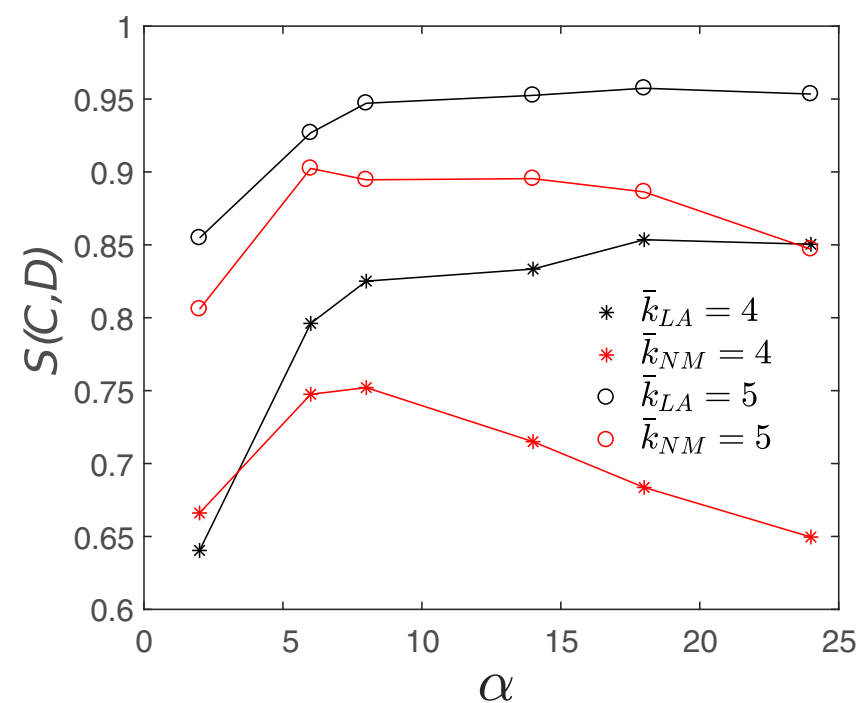

FIG. 8. Dependence of $S\left(C, D_{\mathrm{NM}}\right)$ and $S\left(C, D_{\mathrm{LA}}\right)$ on $\alpha$ is qualitatively equivalent for both algorithms. Red symbols and lines correspond to Newman algorithm, black symbols represent from Louvain. Stars: $\bar{k}=4$, Circles, $\bar{k}=5$. Other parameters in both cases $n=10, N=3, M=20$.

it remains to demonstrate whether it is able to truly discover hidden structures in complex communities in terms of identifying multiple LUs within a single community. To this end, we generate a synthetic network with different communities containing different number of LUs (Fig. 9) and evaluate the capability of our method to detect all LUs accurately. Similar to the previous investigations, we generate the network $G(V, E)$ with three LUs, each LU contains $n$ nodes with average degree $k$. We generate $M_{1}$ projection nodes as before, with connections to the nodes in the union of $\mathrm{LU}_{1}$, $\mathrm{LU}_{2}$, and $\mathrm{LU}_{3}$. Additionally we generate $M_{2}$ projection nodes connecting with the nodes of the union of $\mathbb{M}_{1}$ and $L_{3}$.

To achieve this we execute the following steps:

(1) Same as in the previous algorithm we generate connected components $\mathrm{LU}_{i}\left(V_{i}, E_{i}\right), i \in\{1, \ldots, 3\}$. In each LU, every node connects with other nodes with an equal probability ( $p, 0<p \leqslant 1$ ). We set $n_{i}=n$ the number of nodes in $\mathrm{LU}_{i}$.

(2) $M_{1}$ new nodes are added as projection nodes. $\mathbb{M}_{1}$ is the set of these nodes. For every new node $j \in\left\{1, \ldots, M_{1}\right\}$, $m_{j}$ edges are generated, where $m_{j}$ is drawn from the uniform distribution on $\left[a_{1}, a_{2}\right]$ with $a_{2} \geqslant a_{1} \geqslant 2$. Each edge connects with a random node in the union of $\mathrm{LU}_{1}, \mathrm{LU}_{2}$ with a probability $p_{1}\left(0.5<p_{1}<1\right)$ or with a random node in $\mathrm{LU}_{3}$ with probability $\left(1-p_{1}\right)$. Thus, if $p_{1}$ is large, these projection nodes unite two LUs into one community. In addition, these $M_{1}$ projection nodes connect with each other randomly with a connection probability $p_{2}\left(0<p_{2} \leqslant 1\right)$.

(3) Add $M_{2}$ new nodes, $\mathbb{M}_{2}$ indicates the set of these nodes. The nodes from $\mathbb{M}_{2}$ connect with the nodes in a union of $\mathrm{LU}_{3}$ and $\mathbb{M}_{1}$ randomly. Each new node $q \in\left\{1, \ldots, M_{2}\right\}$, has $h_{q}$ edges. We take $h_{q}$ to be independently generated from the uniform distribution on $\left[b_{1}, b_{2}\right]$ with $b_{2} \geqslant b_{1} \geqslant 2$.

Our method accurately detects multiple LUs within communities of different size (Fig. 10). This is a critical feature of

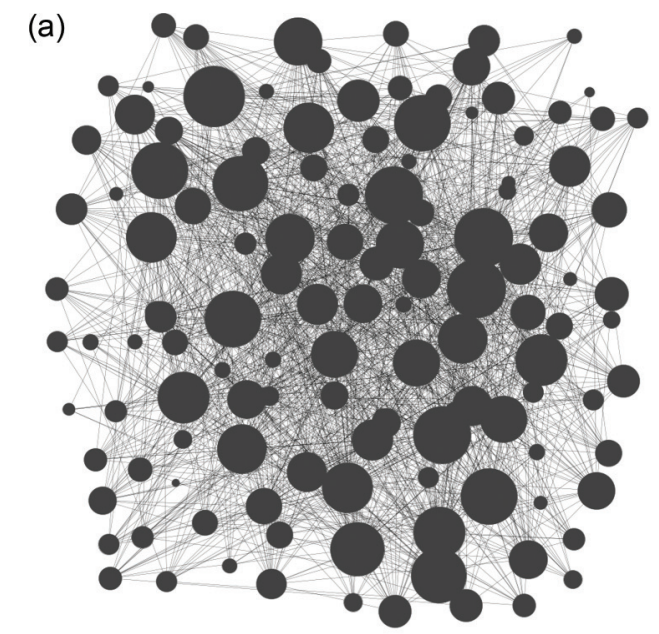

(b)

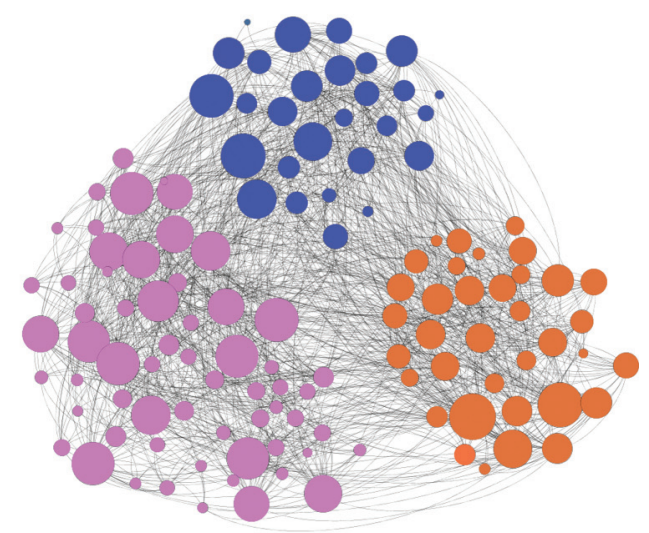

(c)

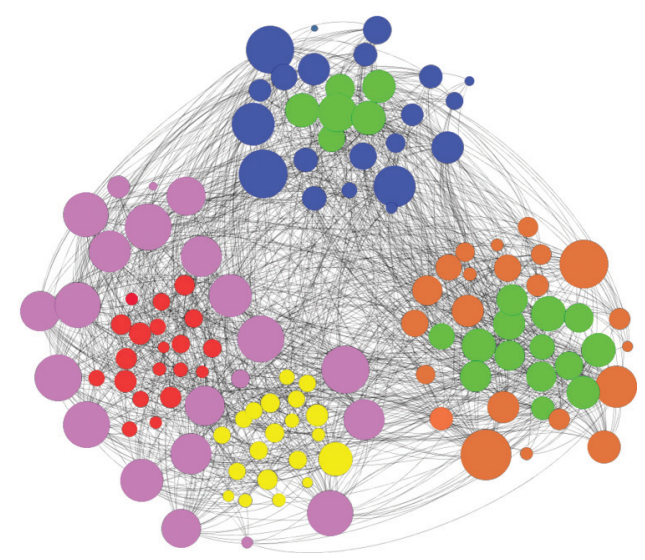

FIG. 9. A synthetic network with different number of LUs per community. (a) Synthetic network created by parameters: $n=20$, $N=3, p=0.2, a_{1}=a_{2}=20, b_{1}=2, b_{2}=30, M_{1}=25, M_{2}=$ $40, p_{1}=0.7$, and $p_{2}=0.2$; (b) communities within the network; (c) local integrator units within each community.

our approach as it allows to zoom into the communities and discover potentially hidden structures that may play a deciding role in the integration of information within the networks. This finding is robust with respect to the choice of community detection algorithm as both Louvain and Newman algorithms 


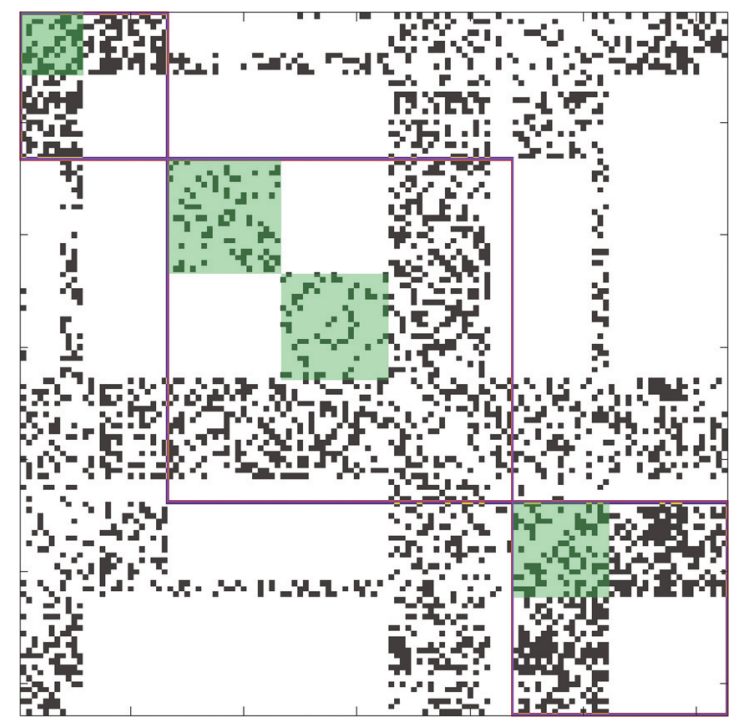

FIG. 10. Detecting communities (violet border) and their local integrator units (green shaded areas) in agreement with the design of the synthetic network.

converge with LA leading to slightly better performance $\left[S\left(C, D_{\mathrm{LA}}\right)=0.95\right.$ and $\left.S\left(C, D_{\mathrm{NM}}\right)=0.85\right]$.

\section{G. Performance evaluation for directed networks}

Natural and technological networks often contain asymmetric interactions that underly the flow of information in these structures [48]. Thus, it is critical to evaluate and compare the performance of our algorithm for directed and undirected networks. To this end, we first generated a synthetic network $G$ (as described in Sec. III A) and assigned a probability measure $p_{\text {dir }}\left(0 \leqslant p_{\text {dir }} \leqslant 1\right)$ to each edge $e_{i j}$ of the network. If adjacency matrix component $A_{i j}$ of $e_{i j}$ is nonzero, then an associated directed network $G_{\mathrm{dir}}$ is given by an adjacency matrix $A^{d}$ such that $A_{i j}^{d}=1$ and with a probability of $p_{\text {dir }}$ and $A_{j i}^{d}=1$ with a probability of $1-p_{\text {dir }}$. By choosing $p_{\text {dir }}=0.5$ we assure the same probability for each edge to be represented in the directed graph as an incoming or outgoing projection or bidirectional connection. Therefore, we conducted the performance tests on an example of $G_{\text {dir }}$ using $p_{\text {dir }}=0.5$ (Fig. 11).

Analogous to our previous tests, we investigated the dependence of $S(C, D)$ on the average LU degree $(\bar{k})$, while increasing the number of local units $(N)$ in the network. Since directionality may affect the community detection process, LU detection was conducted both using Louvain algorithm (Fig. 12) and Newman algorithm (Fig. 13). A direct comparison of $S(C, D)$ curves suggests that our algorithm performs more accurately for undirected networks (black curves) than directed counterparts (red curves). Nonetheless, the general behavior of the algorithm is qualitatively invariant. The LU detection accuracy is in both cases directly proportional to the number of LUs $(N)$ in the network.

The superior performance of our algorithm for undirected networks is best observed in the evaluation of $S(C, D)$ as a function of $\alpha$, thus average projection node degree. Independent of the choice of community detection techniques
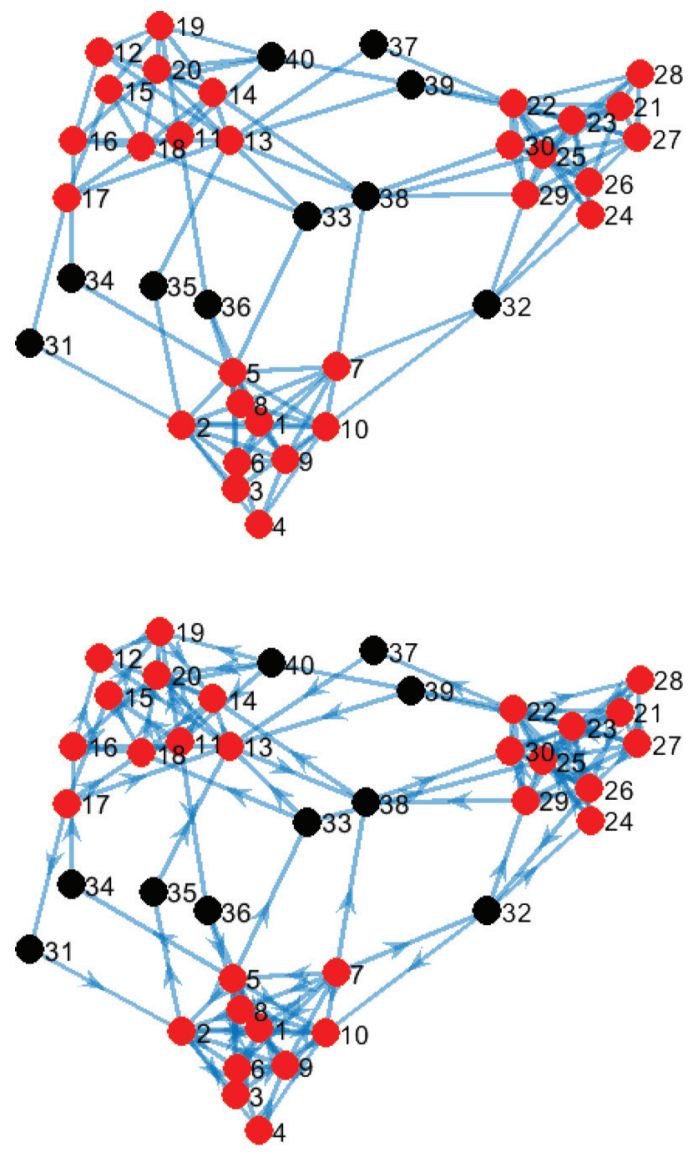

FIG. 11. An undirected network $G$ in which three LUs are in Red and the corresponding directed network $G_{\mathrm{dir}}$ using $p_{\mathrm{dir}}=0.5$.

(Fig. 14 using Louvain algorithm and Fig. 15 using Newman algorithm), $S(C, D)$ if for all $\alpha$ and all tested average degree $\bar{k} \in\{4,5\}$, larger for undirected networks.

\section{LU DETECTION IN C. ELEGANS}

\section{A. C. elegans connectome}

The self-fertilizing hermaphrodite $C$. elegans has 302 neurons, 5000 chemical synapses, 600 gap junctions, and 2000 neuromuscular junctions [14]. Despite its small number of neurons, the worm also shows remarkable behavioral plasticity (e.g., tap habituation [49]) and the capacity to learn and memorize environmental features (e.g., to predict the existence and quality of food [50]). Moreover, the main neurotransmitters in higher animals, such as glutamate, acetylcholine, and GABA, all occur in the nervous system of $C$. elegans. We base our analysis on the data available at Ref. [51] The data represent whole, self-consistent gap junction and chemical synapse networks of hermaphrodite C. elegans [14]. The $C$. elegans network contains 280 nonpharyngeal neurons with 6393 chemical synapses, 890 electrical junctions, and 1410 neuromuscular junctions. To avoid detection of LUs comprising different tissue (e.g., neurons and muscle cells), we exclude $V C 06$ [52], a motor neuron that only contacts a muscle cell. Thus, we consider a neuronal network $N_{W}$ based on the connectivity of 279 neurons that represents the large somatic nervous system. The adjacency matrix is weighted 


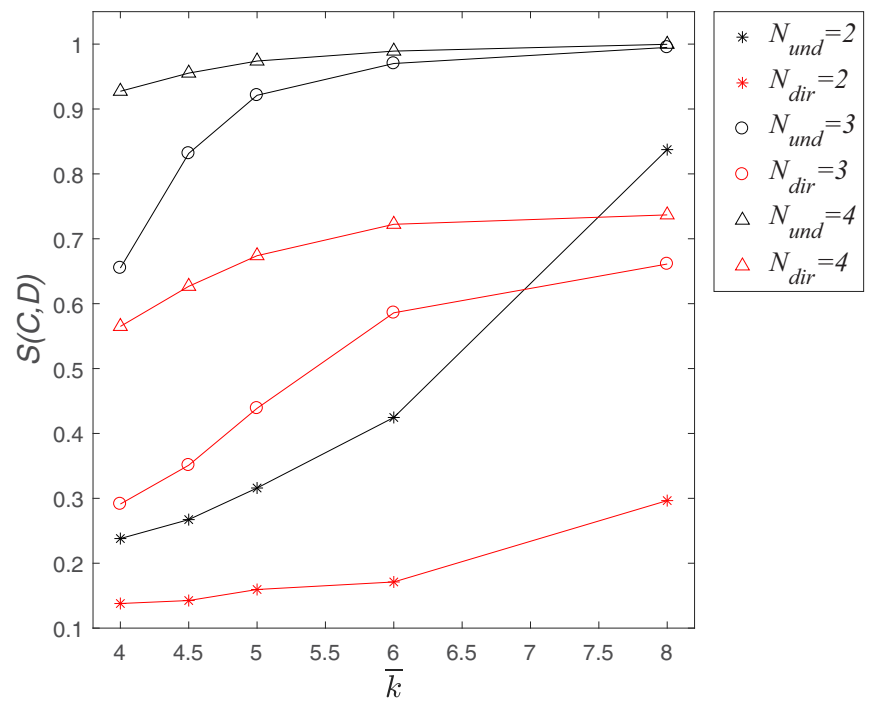

FIG. 12. Dependence of $S(C, D)$ on average degree $\bar{k}$ when communities are detected by Louvain algorithm. Red symbols correspond to the results calculated for directed network $G_{\text {dir }}$, and black symbols represent the results obtained for undirected network $G$. Stars: $N=2$, circles: $N=3$, triangles: $N=4$. Other parameters in both cases are $n=20, \alpha=10, M=30$.

by the number of chemical synapses and is considered undirected. The results of the LU detection algorithm applied to the $C$. elegans neuronal network are presented in Table I.

\section{B. Local integrator units within communities of $C$. elegans connectome}

Overall, we obtain 6 LUs comprising 88 neurons. The largest LU contains 23 INs, and the smallest consists of 4 INs.

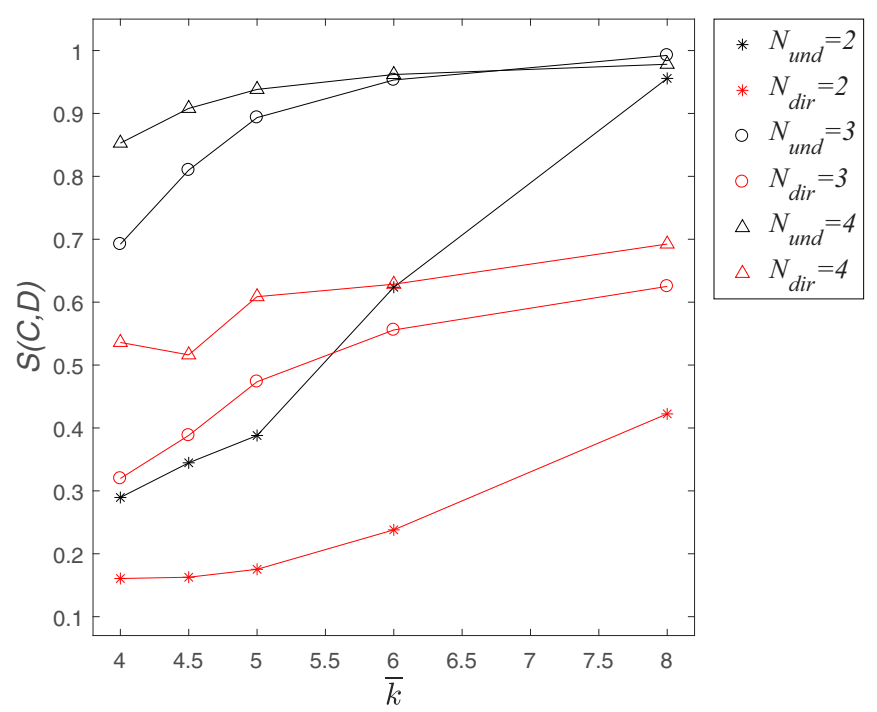

FIG. 13. Dependence of $S(C, D)$ on average degree $\bar{k}$ when communities are detected by Newman method. Red symbols correspond to the results calculated for directed network $G_{\text {dir }}$, and black symbols represent the results obtained for undirected network $G$. Stars: $N=$ 2, circles: $N=3$, triangles: $N=4$. Other parameters in both cases are $n=20, \alpha=10, M=30$.

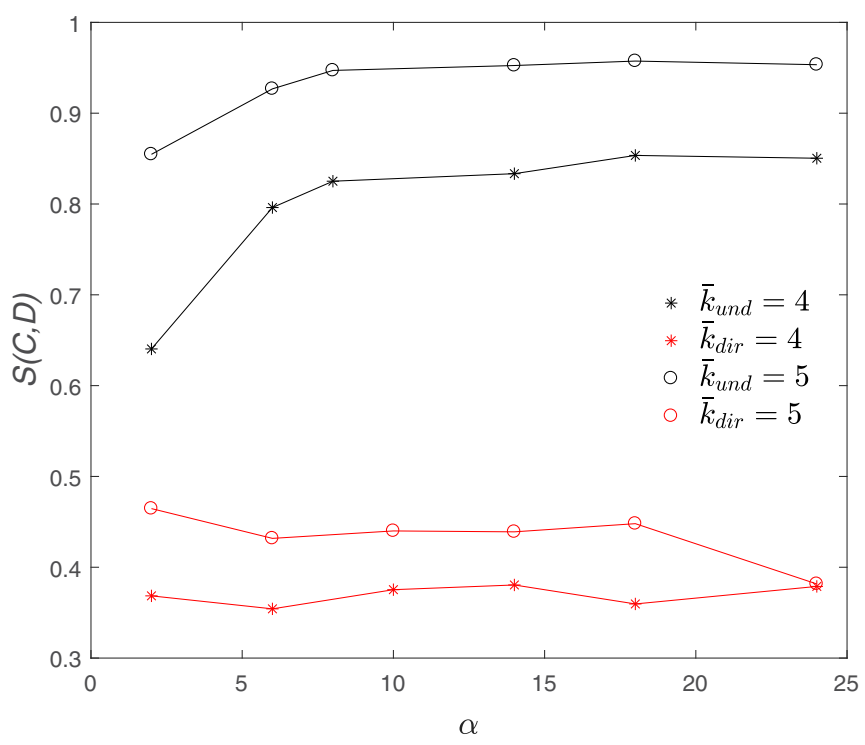

FIG. 14. Dependence of $S(C, D)$ on $\alpha$ when communities are detected by Louvain algorithm. Red symbols correspond to the results calculated for directed network $G_{\text {dir }}$, and black symbols represent the results obtained for undirected network $G$. Stars: $\bar{k}=4$, circles: $\bar{k}=5$. Other parameters in both cases are $n=10, N=3, M=20$.

Most of these LUs are well localized within the worm's body, although no locational information is given to the algorithm. LUs are found in different parts of the C. elegans body: two LUs are in the head approaching the mouth, one is in the neck, one is in the tail, and the other two are near the vulva and anus, respectively. The positions of each LU within the worm body and their neuronal connectivity are shown in Fig. 16. It should also be noted that very few PNs are retained (highlighted in green in Fig. 16). It is noteworthy that the

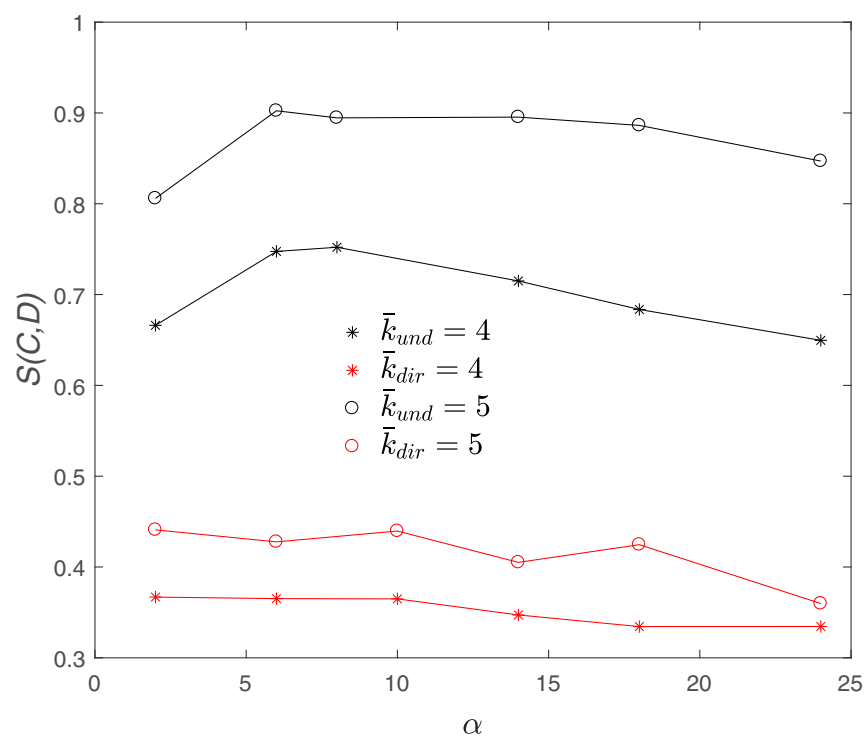

FIG. 15. Dependence of $S(C, D)$ on $\alpha$ when communities are detected by Newman method. Red symbols correspond to the results calculated for directed network $G_{\mathrm{dir}}$, and black symbols represent the results obtained for undirected network $G$. Stars: $\bar{k}=4$, circles: $\bar{k}=5$. Other parameters in both cases are $n=10, N=3, M=20$. 
TABLE I. Detected LUs associated with their anatomical location and physiological function.

\begin{tabular}{|c|c|c|c|c|}
\hline LU & Location & Number of neurons & Neuron functions & References \\
\hline $\mathrm{LU}_{1}$ & Head & 18 & $\begin{array}{l}\text { Chemotaxis, } \\
\text { thermotaxis, locomotion }\end{array}$ & [53-57] \\
\hline $\mathrm{LU}_{2}$ & Head & 23 & $\begin{array}{l}\text { Mechanosensation in the head, } \\
\text { head-withdrawal reflex }\end{array}$ & {$[55,58-60]$} \\
\hline $\mathrm{LU}_{3}$ & Neck & 4 & $\begin{array}{l}\text { locomotion, and } \\
\text { sinusoidal body movement-locomotion }\end{array}$ & {$[61-63]$} \\
\hline $\mathrm{LU}_{4}$ & Vulva & 13 & $\begin{array}{l}\text { Locomotion } \\
\text { and proprioception }\end{array}$ & {$[61-65]$} \\
\hline $\mathrm{LU}_{5}$ & Anus & 8 & $\begin{array}{l}\text { Locomotion, } \\
\text { sinusoidal body movement-locomotion }\end{array}$ & {$[61,63,66]$} \\
\hline $\mathrm{LU}_{6}$ & Tail & 22 & $\begin{array}{l}\text { Locomotion, innervating posterior } \\
\text { dorsal body wall muscles }\end{array}$ & {$[15,61-63,65,67]$} \\
\hline
\end{tabular}

PNs are not localized but stretch to the whole body. Figure 17 shows the correlation between LUs and communities, which are determined based on the initial topology of the $C$. elegans neural network. Seven communities, $C_{1}$ to $C_{7}$, are obtained and are arranged from the top-left to the bottom-right corner. The community boundaries are highlighted by red lines. It can be concluded that each community, except $C_{4}$, contains a single LU. Precisely, $C_{1}$ contains only $\mathrm{LU}_{1}, C_{2}$ contains only $\mathrm{LU}_{4}, C_{3}$ contains $\mathrm{LU}_{6}$ and the two PN (PVDL and PVDR [68]) of $\mathrm{LU}_{5}, C_{5}$ contains only $\mathrm{LU}_{5}$ with all its INs, $C_{6}$ contains only $\mathrm{LU}_{2}$, and $C_{7}$ contains only $\mathrm{LU}_{3} . C_{4}$ does not contain any LU. This observation further supports the advantages of LU detection over the identification of community structure to understand anatomy-function relationships. The main advantage of our method lies in its ability to detect neuronal subnetworks with meaningful physiological functions. Therefore, we screened the existing literature for information on the function of different neuron types that are contained in respective LUs to estimate the potential function of detected LUs. We successfully demonstrate the appropriateness of our algorithm by three examples:

Example $1-L U_{1}$. $\mathrm{LU}_{1}$ contains $16 \mathrm{INs}$ and $2 \mathrm{PNs}$ (PVQL and PVQR [69]), which is localized approaching the mouth. With the exception of neurons ASJL and ASJR [70], all INs have either chemotaxis or thermotaxis functions that are closely related to cognition behavior in C. elegans. It is hypothesized that $\mathrm{LU}_{1}$ is a special structure that mainly processes the cognition function.

Example 2- $\mathrm{LU}_{6}$. Another interesting LU finding is related to $\mathrm{LU}_{6}$, which includes the neuron PDB [15]. In $\mathrm{LU}_{6}$, half of the neurons are from DA, DB, DD, VA, VB, and VD [61-63,65,67], which mainly process locomotion function in C. elegans. In addition, neuron AS11 has been suggested as a processing locomotion function [16]. Therefore, a likely hypothesis would be that PDB is also important for locomotion. Interestingly, a recent interdisciplinary study [33] extensively demonstrated that PDB also has a locomotion function. This example suggests that our LU partitions may provide a reliable alternative for generating hypotheses on the role of cells within neuronal networks.

Example 3-DD neurons. DD neurons [71] are mainly processing locomotion function in C. elegans. A recent study [33] demonstrated that DD05 and DD06 affected posterior body movement. Our results demonstrate DD02 and DD03 in $\mathrm{LU}_{4}, \mathrm{DD} 05$ in $\mathrm{LU}_{5}$, and DD06 in $\mathrm{LU}_{6}$. Therefore, we can understand the relevant result in Ref. [33] that DD05 and DD06 are in the LUs near the anus and tail, so they have observable effects on posterior body movement.

Hereby, it is critical to note that the relationship between LU and respective community should be viewed in a strictly

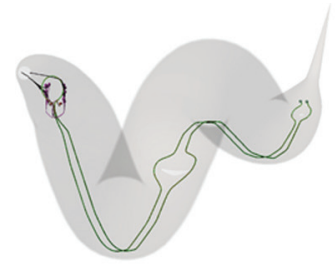

LU1

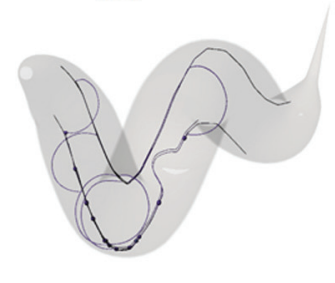

LU4

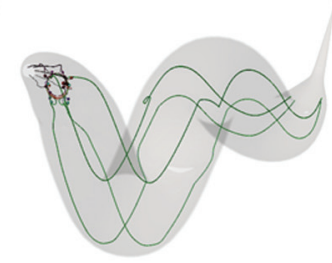

LU2

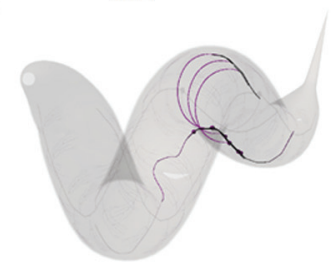

LU5

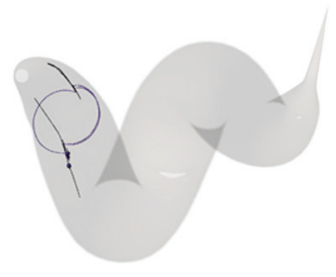

LU3

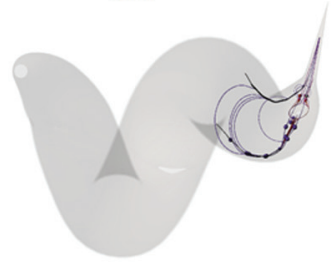

LU6

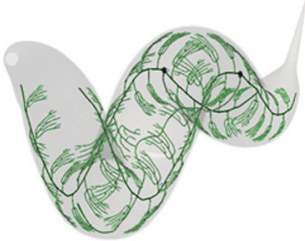

Two PNs in LU5

FIG. 16. Neuron connectivity in each LU in C. elegans nervous system. Six LUs are detected, and the PNs are highlighted in green. 


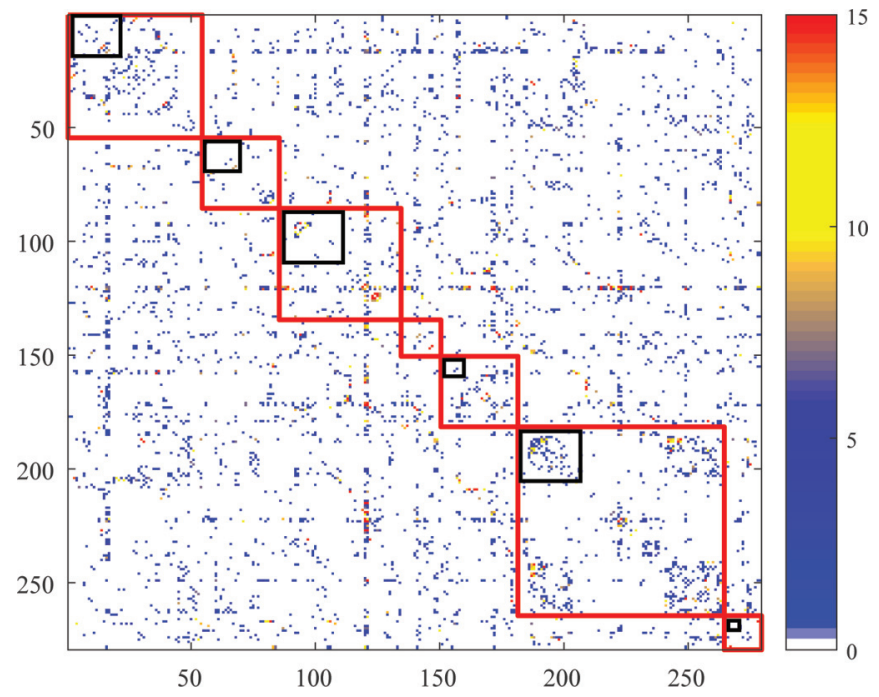

FIG. 17. A community partition of the C. elegans neural network is presented on the adjacency matrix. A larger number on the color bar represents a stronger connection between a pair of nodes. The coloring of the matrix elements reflects the weight assigned to the edges. The relations between initial community divisions and LUs are as follows: $C_{1}$ contains $\mathrm{LU}_{1} ; C_{2}$ includes $\mathrm{LU}_{4} ; C_{3}$ contains $\mathrm{LU}_{6}$ and two PNs of $\mathrm{LU}_{5} ; C_{4}$ does not contain any $\mathrm{LU} ; C_{5}$ includes $\mathrm{LU}_{5}$ with all its INs; $C_{6}$ includes $\mathrm{LU}_{2}$; and $C_{7}$ contains $\mathrm{LU}_{3}$. The red boxes indicate communities within the connectome. The black boxes represent local units within the communities.

mathematical form. It means that $\mathrm{LU}_{i}$ is a subset but not a proper subset of the community $C_{i}$. In other words, there can always be situations where $\mathrm{LU}=C$. This case by itself is a meaningful finding as it shows the lack of further refined structure within a community. While not for $C$. elegans (due to the rather small network size), our tests on synthetic networks further suggest that some communities may have more than one LU which can be detected by our algorithm. Therefore, we can conclude the following information from the algorithm: If a nontrivial $\mathrm{LU}$ (i.e., $\mathrm{LU}_{i}$ proper subset of $C_{i}$ ) is detected, then we gain information on internal structure within $C_{i} . \mathrm{LU}_{i}=C_{i}$ in turn indicates that for $C_{i}$ no additional intrinsic features can be detected than those already revealed by community detection.

While the algorithm detects 6 local integrator units within C. elegans network, it is critical to estimate the reliability of this finding in terms of algorithmic performance $S(C, D)$. To this end, we generated an undirected synthetic network "model" using the network parameters of $C$. elegans connectome $(N=6, k=7, \alpha=10, n=15$ (88 interneurons distributed among 6 LUs) and $M=191)$ and ran the algorithm 100 times to obtain the distribution of $S(C, D)$. The performance ranges between 0.75 and 1 , with a mean at 0.9559 (Fig. 18) indicating a high accuracy of the algorithm for $C$. elegans network.

\section{LIMITATIONS}

As shown above, LUs are detected by removing nodes with a high participation coefficient $P$. However, there are cases that might lead to errors in preserving PNs and deleting INs instead during the process. In particular, LU nodes having

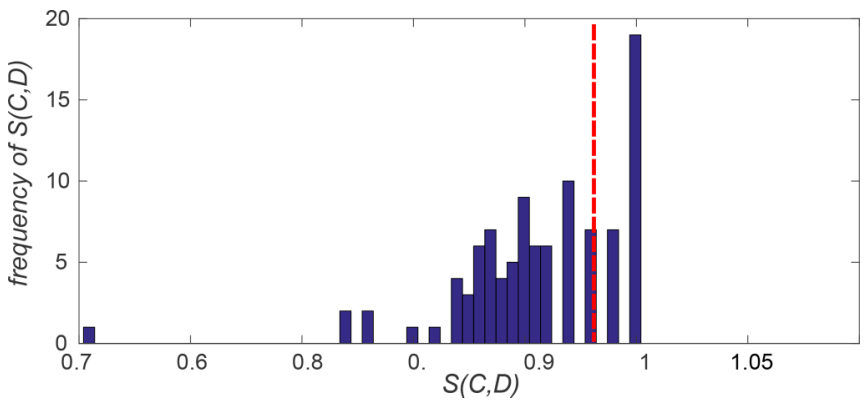

FIG. 18. Histogram of $S(C, D)$ calculated for a synthetic network model of $C$. elegans connectome, red dashed line marks the average $S(C, D)=0.956$.

connections with projection nodes may have a positive $P$. Thus, some INs with the highest $P$ may be taken as PNs and removed. Thereby, during the process of community division, INs and PNs will be grouped into the same community with a large probability if they are strongly connected. Consequently, such PNs are preserved, and instead INs connecting to the PNs will wrongfully be deleted, e.g., two PNs in $\mathrm{LU}_{1}$, four PNs in $\mathrm{LU}_{2}$, and two PNs in $\mathrm{LU}_{5}$ (Fig. 16). To preserve more neurons in LUs, one can relax the threshold on the participation index, allowing $\left\{P_{i} \leqslant \Theta\right\}, \Theta>0$, for an appropriate $\Theta>0$. Alternatively, if the community structure is very distinctive $(Q \geqslant 0.3)$ [72], then one can create a subnetwork based on the connectivity in each community and then apply our model directly to these subnetworks. For example, it can be shown that two more neurons (ASEL and AWCL) are contained in $\mathrm{LU}_{1}$, and four more neurons (DB01, DD01, VA01, and VD01) are included in $\mathrm{LU}_{3}$ when $\left\{P_{i} \leqslant \Theta\right\}$ with $\Theta=0.2$.

As mentioned above, the choice of Louvain algorithm in our method to detect the communities is not unique. LA is a stochastic method and allows a rapid performance; however, we also observe a small difference in the communities depending on the realization. Nonetheless, extensive numerical experiments suggest that LUs with distinct features can be robustly detected in all LUs.

We tested the performance of our algorithm on synthetic directed networks and also applied our model to the directed weighted network of neuron connectivity in the $C$. elegans system. Our analysis suggests that our algorithm performs better and is best applied on undirected graphs. In general, the structures we wish to detect (LUs) are defined actually independent on directionality (the same ground-truth LUs will be there in both directed and undirected synthetic networks). Thus, directionality does not provide additional insights, yet it may induce noise that can be detrimental for the performance of the algorithm, in particular through an underestimation of participation coefficients. Furthermore, in many coupled systems, it is difficult to estimate the directed coupling strength with high reliability. Therefore, we propose adopting the undirected network to detect LUs.

The evaluation of algorithms often depends on the properties and, in particular, the goodness of input data. To evaluate the appropriateness of $N_{W}$ as a reliable test model for our algorithm, we transform $N_{W}$ into a binary network and study the similarity between the anatomically established Ganglion division and binary networks (see Appendix A). The adjusted 
rand index (ARI) is used to describe the similarity. In light of potential a priori errors in synapse detection, we also test the effect of noise on community detection (see Appendix B). The outcomes of the topological sensitivity analysis are presented in the supplementary information. Our analysis suggests that our algorithm performs robustly in the presence of noise and topological alterations.

\section{CONCLUSION}

Brain parcellation is a critical approach for decreasing the complexity of a network and gaining insights into the structural organization. In this study, we introduced a methodology beyond classical parcellation strategies to identify LUs that represent the bottom-most layer of the brain hierarchy. Our method certainly does not provide guarantees for the functional significance of each identified LU; however, it allows us to develop and test meaningful hypotheses. With the constant creation of the data on nervous systems of growing complexity, our method could be used to identify and study neuronal functional subunits. Yet, in addition to the neural system, our methodology also provides insights into understanding of complex systems and could be applied extensively to a variety of networks that may possess an LU structure.

The accuracy of the algorithm was successfully demonstrated on synthetic networks, and the sensitivity of its performance with respect to network parameters and choice of community detection algorithms was obtained. A unique feature of our algorithm is that it relies only on network properties and allows a deeper look into the intrinsic features of communities. To verify which of the LUs we observed are most reasonable and significant, one should ideally also use anatomical and cytoarchitectural data. For the case of $C$. elegans, we considered the localization of the neurons within the body as a verification criterion and found that most LUs found by the algorithm were reasonably localized.

In conclusion, our study proposes a method for the network analysis of neuronal systems and other network structures with locality features. By applying this method to the $C$. elegans nervous system, we uncovered valuable information, allowing us to allocate functions of neurons according to their functional unit partition. Our method can particularly be used for hypothesis generation on the yet unknown functions of neurons within neuronal clusters. Ablation or photostimulation experiments can be performed on the neurons from the identified LUs to validate their group function and infer the individual significance of the neurons. For instance, we could indicate the function of PDB and a potentially more comprehensive understanding of some DD neurons, which were only recently characterized experimentally [33].

In addition to the appropriateness of our method for discovering LUs in the C. elegans connectome, the properties of the algorithm, particularly its use of the stochastic LA, make it computationally efficient for larger networks and thus allow its application to connectomes of different sizes $[7,11]$.

\section{ACKNOWLEDGMENTS}

We thank David Hall and his team for supplying the worm template (Albert Einstein College of Medicine, Department of Neuroscience), Stephen D. Larson (Ph.D. at the OpenWorm Foundation) and Christian Grove (at WormBase) for the neuron image support, Diego Sanchez (MPI for Biological Cybernetics) for discussions on blender, and Raymond Lee (at WormBase) for a valuable discussion about ganglion neurons. We thank Maria Ercsey-Ravasz (Babes-Bolyai University, Romania), Chitin Shih (Department of Applied Physics, Tunghai University, Taiwan), and Morteza Soltanpour (MPI for Biological Cybernetics) for valuable discussions. This work was supported by the Bundesministerium für Bildung und Forschung (e:Med program: FKZ: 01ZX1311A [73] and FKZ: 01ZX1503), and the European Union's Horizon 2020 Program 668863-SyBil-AA, National Natural Science Foundation of China (Grant No. 11305017), and Specialized Foundation for Theoretical Physics of China (Grant No. 11247239). A.L. was supported by a Sofja Kovalevskaja award from the Alexander von Humboldt Foundation and by the Institutional Strategy of the University of Tübingen (DFG, ZUK 63).

\section{APPENDIX A: ARI SCORE}

To evaluate whether $N_{W}$ is a reasonable model, we make $N_{W}$ binary and study the similarity between the anatomically established Ganglion division and binary networks. We define a binary network $N_{\mathrm{BI}, \theta}$ by the adjacency matrix $A^{\mathrm{BI}}$ : given a threshold $\theta$,

$$
A_{i j}^{\mathrm{BI}}=\left\{\begin{array}{ll}
0, & \text { if } A_{i j}<\theta \\
1, & \text { otherwise }
\end{array},\right.
$$

where $A_{i j}$ is the entity in adjacency matrix $A$ of $N_{W}$. The average edge weight of nodes in $N_{W}$ is given by $\bar{w}=3.6$; thus, we consider $\theta \in\{1,2,3,4\}$.

Ganglion division is a biological classification consisting of ten groups of neurons (nine groups of ganglions and one ventral cord neuron group) $[14,74,75]$. Here, we utilize the adjusted Rand Index (ARI) [76,77] to measure the similarity between two data clusterings. ARI is a modification of Rand Index [78] that is interpreted as the fraction of vertex (node) pairs that are consistent: A vertex pair is consistent between two partitions if either (a) the vertex pair is within the same group in both partitions or (b) the vertex pair is split between two groups in both partitions. The interpretation of the RI depends on the number of groups [79], whereas ARI is adjusted for chance agreement and number of groups. Though the Rand Index may only yield a value between 0 and +1 , the ARI can yield negative values if the index is less than the expected index. ARI is defined as

$$
\mathrm{ARI}=\frac{\mathrm{RI}-\mathbb{E}(\mathrm{RI})}{\max (\mathrm{RI})-\mathbb{E}(\mathrm{RI})},
$$

where the expectation $E(\mathrm{RI})$ is computed assuming a hypergeometric distribution of the counts of consistent vertex pairs.

Table II shows the ARI score between the Ganglion division and different network models, in which four types of binary network $N_{\mathrm{BI}, \theta}$ are considered. ARI $\mathrm{AR}_{\text {.N }}$ represents

TABLE II. ARI score.

\begin{tabular}{lccccc}
\hline \hline & $N_{W}$ & $N_{\mathrm{BI}}, \theta=1$ & $N_{\mathrm{BI}, \theta=2}$ & $N_{\mathrm{BI}, \theta=3}$ & $N_{\mathrm{BI}, \theta=4}$ \\
\hline ARI $_{G . N}$ & 0.264 & 0.209 & 0.235 & 0.246 & 0.221 \\
$Q$ & 0.509 & 0.411 & 0.461 & 0.519 & 0.561 \\
\hline \hline
\end{tabular}




\begin{tabular}{|l|l|l|l|l|l|}
\hline LU1 & LU2 & LU3 & LU4 & LU5 & LU6 \\
\hline AFDL & CEPDL & AS01 & AS04 & DD05 & AS11 \\
\hline AFDR & CEPDR & DA01 & DA03 & PVDL & DA08 \\
\hline AIAL & L1L & VA02 & DA05 & PVDR & DA09 \\
\hline AIAR & RIAL & VD02 & DB02 & VA09 & DB07 \\
\hline ASER & RIAR & & DD02 & VB08 & DD06 \\
\hline ASGL & RIVL & & DD03 & VB09 & LUAL \\
\hline ASGR & RIVR & & VA04 & VD09 & LUAR \\
\hline ASIL & RMDDL & & VA05 & VD10 & PDA \\
\hline ASIR & RMDDR & & VA06 & & PDB \\
\hline ASJL & RMDL & & VB03 & & PHBL \\
\hline ASJR & RMDR & & VB04 & & PHBR \\
\hline ASKL & RMDVL & & VB05 & & PHCL \\
\hline ASKR & RMDVR & & VB06 & & PHCR \\
\hline AWAL & RMHL & & & & PQR \\
\hline AWAR & SMDDL & & & & PVWL \\
\hline AWCR & SMDDR & & & & PVWR \\
\hline PVQL & SMDVL & & & & VA11 \\
\hline PVQR & SMDVR & & & & VA12 \\
\hline & URBL & & & & VB10 \\
\hline & URYDL & & & & VB11 \\
\hline & URYDR & & & & VD12 \\
\hline & URYVL & & & & VD13 \\
\hline & URVR & & & & \\
\hline
\end{tabular}

FIG. 19. LN list in each LU in the weighted network $N_{W}$. The names of PNs are in bold.

ARI score between ganglion division (G) and partitions of the network $N$ by community detection algorithm (LA). It is seen from Table II that $N_{W}$ obtains the highest score among all the models. Making $N_{W}$ binary may still result in distinct community structures (for example, for $N_{\mathrm{BI}, \theta=3}$ ); nevertheless, parts of valuable information are lost. This study shows that partition in $N_{W}$ is closer to the biological partition in C. elegans nervous system (Fig. 19).

\section{APPENDIX B: ROBUSTNESS OF $N_{W}$}

Considering that there might be some errors in synapse detection, we test the effect of noise on community detection.

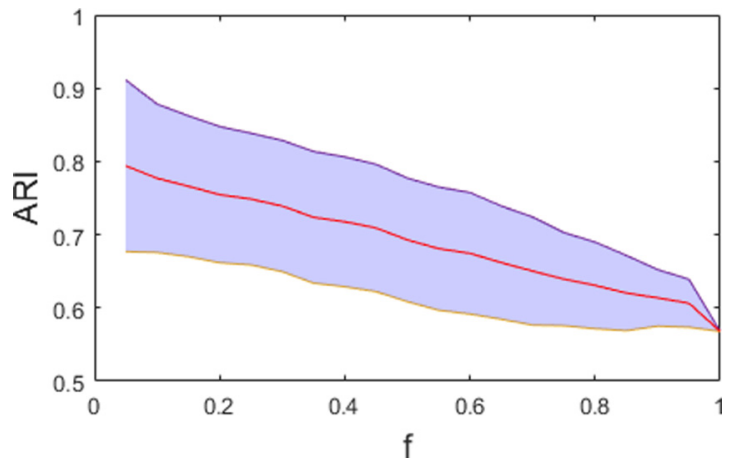

FIG. 20. Variation of ARI with noise strength $f$. ARI measures the similarity between community partition of $N_{W}$ and $N_{W}$, noise when noise is considered in $N_{W}$. All ARI data are obtained by averaging 1000-time calculations and correspond to the dot on the red line. Data on the blue and yellow lines are $\mathrm{ARI} \pm \sigma$ respectively, where $\sigma$ is the standard deviation.

We introduce the noise to each pair of neurons whose connection strength is no less than 1 . Therefore,

$$
A_{i j}^{\text {noise }}= \begin{cases}A_{i j}-1, & \text { if } r<f \\ A_{i j}, & \text { otherwise }\end{cases}
$$

where $f(0 \leqslant f \leqslant 1)$ is the noise strength, and $r$ is a random number. ARI is calculated between $N_{W}$ and models of $N_{W}^{\text {noise }}$ under different noise strength, and the variation of ARI with $f$ is shown in Fig. 20. It is observed that ARI decreases as $f$ increase, and ARI is about 0.6 when $f=1$. It is concluded that $N_{W}$ is generally robust, and many features can be retained even there is a strong noise.
[1] K. Brodmann, Vergleichende Lokalisationslehre der Grosshirnrinde in ihren Prinzipien dargestellt auf Grund des Zellenbaues (Johann Ambrosius Barth Verlag, Leipzig, 1909).

[2] W. Penfield and E. Boldrey, Somatic motor and sensory representation in the cerebral cortex of man as studied by electrical stimulation, Brain 60, 389 (1937).

[3] A. W. Toga, P. M. Thompson, S. Mori, K. Amunts, and K. Zilles, Towards multimodal atlases of the human brain, Nat. Rev. Neurosci. 7, 952 (2006).

[4] K. Amunts and K. Zilles, Architectonic mapping of the human brain beyond brodmann, Neuron 88, 1086 (2015).

[5] M. F. Glasser, T. S. Coalson, E. C. Robinson, C. D. Hacker, J. Harwell, E. Yacoub et al., A multimodal parcellation of human cerebral cortex, Nature 536, 171 (2016).

[6] O. Sporns, G. Tononi, and R. Kötter, The human connectome: A structural description of the human brain, PLoS Comput. Biol. 1, e42 (2005).

[7] S. W. Oh, J. A. Harris, L. Ng, B. Winslow, N. Cain, S. Mihalas, and M. T. Mortrud, A mesoscale connectome of the mouse brain, Nature 508, 207 (2014).
[8] D. C. Van Essen, J. H. R. Maunsell, and J. L. Bixby, The middle temporal visual area in the macaque: Myeloarchitecture, connections, functional properties, and topographic organization, J. Comp. Neurol. 199, 293 (1981).

[9] J. L. Vincent, G. H. Patel, M. D. Fox, A. Z. Snyder, J. T. Baker, D. C. Van Essen, J. M. Zempel, L. H. Snyder, M. Corbetta, and M. E. Raichle, Intrinsic functional architecture in the anaesthetized monkey brain, Nature 447, 83 (2007).

[10] C. Van Essen David, C. Donahue, D. L. Dierker, and M. F. Glasser, Parcellations and connectivity patterns in human and macaque cerebral cortex, in Research and Perspectives in Neurosciences, Micro-, Meso-, and Macro-connectomics of the Brain (Springer, Berlin, 2016), pp. 89-106.

[11] H. R. Noori, J. Schöttler, M. Ercsey-Ravasz, A. Cosa-Linan, M. Varga, Z. Toroczkai et al., A multiscale cerebral neurochemical connectome of the rat brain, PLoS Biol. 15, e2002612 (2017).

[12] A. S. Chiang, C. Y. Lin, C. C. Chuang, H. M. Chang, C. H. Hsieh, C. W. Yeh, C. T. Shih, J. J. Wu, G. T. Wang, Y. C. Chen 
et al., Three dimensional reconstruction of brain-wide wiring networks in Drosophila at single-cell resolution, Curr. Biol. 21, 1 (2011).

[13] C. T. Shih, O. Sporns, S. L. Yuan, T. S. Su et al., Connectomicsbased analysis of information flow in the Drosophila brain, Curr. Biol. 25, 1249 (2015).

[14] L. R. Varshney, B. L. Chen, E. Paniagua, D. H. Hall, and D. B. Chklovskii, Structural properties of the Caenorhabditis elegans neuronal network, PLoS Comput. Biol. 7, e1001066 (2011).

[15] T. A. Jarrell, Yi Wang, A. E. Bloniarz, C. A. Brittin, M. $\mathrm{Xu}$, J. Nichol Thomson, D. G. Albertson, D. H. Hall, and S. W. Emmons, The connectome of a decision-making neural network, Science 337, 437 (2012).

[16] Z. F. Altun, L. A. Herndon, C. A. Wolkow, C. Crocker, R. Lints, and D. H. Hall (eds.) WormAtlas, 2002-2017, retrieved from http://www.wormatlas.org/neuronalwiring.html.

[17] K. Rhrissorrakrai and K. C. Gunsalus, MINE: Module identification in networks, BMC Bioinf. 12, 4581 (2011).

[18] A. Arenas, A. Fernandez, S. Gömez, A Complex Network Approach to the Determination of Functional Groups in the Neural System of C. Elegans, BIOWIRE 2007: Bio-Inspired Computing and Communication, Lecture Notes in Computer Science (Springer, Berlin, Heidelberg, 2008), Vol. 5151, pp. $9-18$.

[19] E. Bullmore and O. Sporns, Complex brain networks: Graph theoretical analysis of structural and functional systems, Nature Rev. Neurosci. 10, 186 (2009).

[20] M. P. van den Heuvel, L. H. Scholtens, and M. A. de Reus, Topological organization of connectivity strength in the rat connectome, Brain Struct. Funct. 221, 1719 (2016).

[21] A. Stocco, C. Lebiere, and J. R. Anderson, Conditional routing of information to the cortex: A model of the basal ganglia's role in cognitive coordination, Psychol. Rev. 117, 541 (2010).

[22] J. P. Bolam, J. J. Hanley, P. A. Booth, and M. D. Bevan, Synaptic organisation of the basal ganglia, J. Anat. 196, 527 (2000).

[23] J. M. Tepper and J. P. Bolam, Functional diversity and specificity of neostriatal interneurons, Curr. Opin. Neurobiol. 14, 685 (2004).

[24] J. G. White, E. Southgate, J. N. Thomson, and S. Brenner, The structure of the nervous system of the nematode Caenorhabditis elegans, Philos. Trans. R. Soc. London B 314, 1 (1986).

[25] K. Ryan, Z. Lu, and I. A. Meinertzhagen, The CNS connectome of a tadpole larva of Ciona intestinalis (L.) highlights sidedness in the brain of a chordate sibling, Elife 5, e16962 (2016).

[26] E. K. Towlson, P. E. Vértes, S. E. Ahnert, W. R. Schafer, and E. T. Bullmore, The rich club of the C. elegans neuronal connectome, J. Neurosci. 33, 6380 (2013).

[27] K. A. Bacik, M. T. Schaub, M. Beguerisse-Díaz, Y. N. Billeh, and M. Barahona, Flow-based network analysis of the Caenorhabditis elegans connectome, PLoS Comput. Biol. 12, e1005055 (2016).

[28] A. Azulay, E. Itskovits, and A. Zaslaver, The C. elegans connectome consists of homogenous circuits with defined functional roles, PLoS Comput. Biol. 12, e1005021 (2016).

[29] S. Kim, H. Kim, J. D. Kralik, and J. Jeong, Vulnerability-based critical neurons, synapses, and pathways in the Caenorhabditis elegans connectome, PLoS Comput. Biol. 12, e1005084 (2016).

[30] T. Huang, Y. Sun, Z. Zhang, S. Deng, and R. Peng, Monoamine and neuropeptide connections significantly alter the degree distributions of the Caenorhabditis elegans connectome, NeuroReport 28, 1071 (2017).

[31] B. L. Chen, D. H. Hall, and D. B. Chklovskii, Wiring optimization can relate neuronal structure and function, Proc. Natl. Acad. Sci. USA 103, 4723 (2006).

[32] A. R. Benson, D. F. Gleich, and J. Leskovec, Higherorder organization of complex networks, Science 353, 163 (2016).

[33] G. Yan, P. E. Vértes, E. K. Towlson, Y. L. Chew, D. S. Walker, W. R. Schafer, and A. L. Barabási, Network control principles predict neuron function in the Caenorhabditis elegans connectome, Nature 550, 519 (2017).

[34] R. Guimerà and L. A. Nunes Amaral, Functional cartography of complex metabolic networks, Nature 433, 895 (2005).

[35] V. D. Blondel, J. L. Guillaume, R. Lambiotte, and E. Lefebvre, Fast unfolding of communities in large networks, J. Stat. Mech. (2008) P10008.

[36] M. E. Newman, Finding community structure in networks using the eigenvectors of matrices, Phys. Rev. E 74, 036104 (2006).

[37] M. Newman and M. Girvan, Finding and evaluating community structure in networks, Phys. Rev. E 69, 026113 (2004).

[38] M. Newman, Modularity and community structure in networks, Proc. Natl. Acad. Sci. USA 103, 8577 (2006).

[39] M. Rubinov and O. Sporns, Complex network measures of brain connectivity: Uses and interpretations, Neuroimage 52, 1059 (2010).

[40] M. Girvan and M. Newman, Community structure in social and biological networks. Proc. Natl. Acad. Sci. USA 99, 7821 (2002).

[41] A. Pothen, Graph partitioning algorithms with applications to scientific computing, in Parallel Numerical Algorithms (Springer, Dordrecht, 1997), pp. 323-368.

[42] B. W. Kernighan and S. Lin, An efficient heuristic procedure for partitioning graphs, Bell Syst. Tech. J. 49, 291 (1970).

[43] E. R. Barnes, An algorithm for partitioning the nodes of a graph, SIAM J. Algebr. Discrete Methods 3, 541 (1982).

[44] T. Hastie, R. Tibshirani, and J. H. Friedman, The Elements of Statistical Learning (Springer, Berlin, Germany, 2001).

[45] J. MacQueen, Some methods for classification and analysis of multivariate observations, in Proceedings of the 5th Berkeley Symposium on Mathematical Statistics and Probability (University of California Press, US, 1967), Vol. 1, pp. 281-297.

[46] J. C. Bezdek, Objective function clustering, in Pattern Recognition with Fuzzy Objective Function Algorithms (Springer, Boston, MA, 1981), pp. 43-93.

[47] U. Von Luxburg, A tutorial on spectral clustering, Stat. Comput. 17, 395 (2007).

[48] K. Klemm and S. Borholdt, Topology of biological networks and reliability of information processing, Proc. Natl. Acad. Sci. USA 102, 18414 (2005).

[49] J. K. Rose and C. H. Rankin, Analyses of habituation in Caenorhabditis elegans, Learn. Memory 8, 63 (2001).

[50] E. L. Ardiel and C. H. Rankin, An elegant mind: Learning and memory in Caenorhabditis elegans, Learn. Mem. 17, 191 (2010).

[51] http://www.wormatlas.org/neuronalwiring.html.

[52] C. Li and M. Chalfie, Organogenesis in C. elegans: Positioning of neurons and muscles in the egg-laying system, Neuron 4, 681 (1990). 
[53] C. I. Bargmann and H. R. Horvitz, Chemosensory neurons with overlapping functions direct chemotaxis to multiple chemicals in C. elegans, Neuron 7, 729 (1991).

[54] I. Mori and Y. Ohshima, Neural regulation of thermotaxis in Caenorhabditis elegans, Nature 376, 344 (1995).

[55] J. M. Gray, J. J. Hill, and C. I. Bargmann, A circuit for navigation in Caenorhabditis elegans, Proc. Natl. Acad. Sci. USA 102, 3184 (2005).

[56] Y. Shinkai, Y. Yamamoto, M. Fujiwara, T. Tabata, T. Murayama, T. Hirotsu, and I. Katsura, Behavioral choice between conflicting alternatives is regulated by a receptor guanylyl cyclase, GCY-28, and a receptor tyrosine kinase, SCD-2, in AIA interneurons of Caenorhabditis elegans, J. Neurosci. 31, 3007 (2011).

[57] M. Tomioka, T. Adachi, H. Suzuki, H. Kunitomo, W. R. Schafer, and Y. Iino, The insulin/PI 3-kinase pathway regulates salt chemotaxis learning in Caenorhabditis elegans, Neuron 51, 613 (2006).

[58] L. Kang, J. Gao, W. R. Schafer, Z. Xie, and X. S. Xu, C. elegans TRP family protein TRP-4 is a pore-forming subunit of a native mechanotransduction channel, Neuron 67, 381 (2010).

[59] A. C. Hart, S. Sims, and J. M. Kaplan, Synaptic code for sensory modalities revealed by $C$. elegans GLR-1 glutamate receptor, Nature 378, 82 (1995).

[60] K. S. Kindt, V. Viswanath, L. Macpherson, K. Quast, H. Hu, A. Patapoutian, and W. R. Schafer, Caenorhabditis elegans TRPA-1 functions in mechanosensation, Nat. Neurosci. 10, 568 (2007).

[61] M. Driscoll and J. Kaplan, Mechanotransduction, in C. elegans II, edited by D. L. Riddle, T. Blumenthal, B. J. Meyer, J. R. Priess (Cold Spring Harbor Laboratory Press, Cold Spring Harbor, NY, 1997), pp. 645-677.

[62] N. Tavernarakis, W. Shreffler, S. Wang, and M. Driscoll, unc-8, a DEG/ENaC family member, encodes a subunit of a candidate mechanically gated channel that modulates C. elegans locomotion, Neuron 18, 107 (1997).

[63] Z. F. Altun, B. Chen, Z. W. Wang, and D. H. Hall, High resolution map of Caenorhabditis elegans gap junction proteins, Dev. Dynam. 238, 1936 (2009).

[64] A. Barrios, R. Ghosh, C. Fang, S. W. Emmons, and M. M. Barr, PDF-1 neuropeptide signaling modulates a neural circuit for mate-searching behavior in C. elegans, Nat. Neurosci. 15, 1675 (2012).

[65] Q. Wen, M. D. Po, E. Hulme, S. Chen, X. Liu, S. W. Kwok, M. Gershow, A. M. Leifer, V. Butler, C. Fang-Yen, T. Kawano, W. R. Schafer, G. Whitesides M. Wyart, D. B. Chklovskii, M. Zhen, and A. D. T. Samuel, Proprioceptive coupling within motor neurons drives
C. elegans forward locomotion, Neuron 76, 750 (2012).

[66] J. C. Way and M. Chalfie, The mec-3 gene of Caenorhabditis elegans requires its own product for maintained expression and is expressed in three neuronal cell types, Genes Dev. 3, 1823 (1989).

[67] J. E. Sulston, Post-embryonic development in the ventral cord of Caenorhabditis elegans, Philos. Trans. R. Soc. London B 275, 287 (1976).

[68] D. H. Hall and M. Treinin, How does morphology relate to function in sensory arbors? Trends Neurosci. 34, 443 (2011).

[69] R. M. Durbin, Studies on the development and organisation of the nervous system of Caenorhabditis elegans. Ph.D thesis, University of Cambridge, 1987.

[70] W. S. Schackwitz, T. Inoue, and J. H. Thomas, Chemosensory neurons function in parallel to mediate a pheromone response in C. elegans, Neuron 17, 719 (1996).

[71] J. G. White, D. G. Albertson, and M. A. R. Anness, Connectivity changes in a class of motoneurone during the development of a nematode, Nature 271, 764 (1978).

[72] A. Clauset, M. E. Newman, and C. Moore, Finding community structure in very large networks, Phys. Rev. E 70, 066111 (2004).

[73] R. Spanagel, D. Durstewitz, A. Hansson, A. Heinz, F. Kiefer, G. Köhr, F. Matthäus, M. M. Nöthen, H. R. Noori, K. Obermayer, M. Rietschel, P. Schloss, H. Scholz, G. Schumann, M. Smolka, W. Sommer, V. Vengeliene, H. Walter, W. Wurst, U. S. Zimmermann, Addiction GWAS Resource Group, S. Stringer, Y. Smits, and E. M. Derks, A systems medicine research approach for studying alcohol addiction, Addict. Biol. 18, 883 (2013).

[74] Z. Altun and D. Hall, Handbook of C. elegans anatomy. WormAtlas, https://www.wormatlas.org/handbookhome.htm (2005).

[75] D. M. Pavlovic, P. E. Vértes, E. T. Bullmore, W. R. Schafer, T. E. Nichols, Stochastic blockmodeling of the modules and core of the Caenorhabditis elegans connectome, PLoS ONE 9, e97584 (2014).

[76] J. Handl, J. Knowles, and D. Kell, Computational cluster validation in postgenomic data analysis, Bioinformatics 21, 3201 (2005).

[77] L. Hubert and P. Arabie, Comparing partitions, J. Classif. 2, 193 (1985).

[78] W. M. Rand, Objective criteria for the evaluation of clustering methods, J. Am. Stat. Assoc. 66, 846 (1971).

[79] L. Morey and A. Agresti, The measurement of classification agreement: An adjustment to the rand statistic for chance agreement, Educ. Psychol. Meas. 44, 33 (1984). 